\title{
Enhanced size-dependent piezoelectricity and elasticity in nanostructures due to the flexoelectric effect
}

\author{
M. S. Majdoub, ${ }^{1}$ P. Sharma, ${ }^{1,2, *}$ and T. Cagin ${ }^{3}$ \\ ${ }^{1}$ Department of Mechanical Engineering, University of Houston, Houston, Texas 77204, USA \\ ${ }^{2}$ Department of Physics, University of Houston, Houston, Texas 77204, USA \\ ${ }^{3}$ Department of Chemical Engineering, Texas A\&M University, College Station, Texas 77845, USA
}

(Received 5 November 2007; revised manuscript received 3 January 2008; published 25 March 2008)

\begin{abstract}
Crystalline piezoelectric dielectrics electrically polarize upon application of uniform mechanical strain. Inhomogeneous strain, however, locally breaks inversion symmetry and can potentially polarize even nonpiezoelectric (centrosymmetric) dielectrics. Flexoelectricity - the coupling of strain gradient to polarization-is expected to show a strong size dependency due to the scaling of strain gradients with structural feature size. In this study, using a combination of atomistic and theoretical approaches, we investigate the "effective" sizedependent piezoelectric and elastic behavior of inhomogeneously strained nonpiezoelectric and piezoelectric nanostructures. In particular, to obtain analytical results and tease out physical insights, we analyze a paradigmatic nanoscale cantilever beam. We find that in materials that are intrinsically piezoelectric, the flexoelectricity and piezoelectricity effects do not add linearly and exhibit a nonlinear interaction. The latter leads to a strong size-dependent enhancement of the apparent piezoelectric coefficient resulting in, for example, a "giant" $500 \%$ enhancement over bulk properties in $\mathrm{BaTiO}_{3}$ for a beam thickness of $5 \mathrm{~nm}$. Correspondingly, for nonpiezoelectric materials also, the enhancement is nontrivial (e.g., $80 \%$ for $5 \mathrm{~nm}$ size in paraelectric $\mathrm{BaTiO}_{3}$ phase). Flexoelectricity also modifies the apparent elastic modulus of nanostructures, exhibiting an asymptotic scaling of $1 / h^{2}$, where $h$ is the characteristic feature size. Our major predictions are verified by quantum mechanically derived force-field-based molecular dynamics for two phases (cubic and tetragonal) of $\mathrm{BaTiO}_{3}$.
\end{abstract}

DOI: 10.1103/PhysRevB.77.125424

PACS number(s): 77.65.- j

\section{INTRODUCTION}

In response to mechanical stimuli, certain crystalline dielectrics may electrically polarize. Assuming that the applied uniform mechanical strain $\varepsilon$ is "small enough," empirical evidence and phenomenological considerations suggest the following relation:

$$
(\mathbf{P})_{i}=(\mathbf{d})_{i j k}(\boldsymbol{\varepsilon})_{j k} .
$$

Indices (in some suitable Cartesian framework) are explicitly written to display the order of the matter tensors as prevalently understood in the literature. The third order tensor d is, thus, the piezoelectric matter tensor. Symmetry considerations restrict it be nonzero only for dielectrics belonging to crystallographic point groups that admit noncentrosymmetry. ${ }^{3}$

Centrosymmetric dielectrics evidently are not expected to polarize under mechanical strain. A nonuniform strain field or the presence of strain gradients can, however, locally break inversion symmetry and induce polarization even in centrosymmetric crystals. This phenomenon is termed flexoelectrictiy, ${ }^{4,5}$ inspired by a similar effect in liquid crystals. ${ }^{6-8}$ In a naive approach, we may simply append a term proportional to the strain gradients in Eq. (1):

$$
(\mathbf{P})_{i}=(\mathbf{d})_{i j k}(\boldsymbol{\varepsilon})_{j k}+(\mathbf{f})_{i j k l} \nabla_{l}(\boldsymbol{\varepsilon})_{j k} .
$$

Here, $\mathbf{f}$ is the so-called fourth order flexoelectric tensor. Thus, unlike the components of the third ordered tensor d (piezoelectric coefficients) which are nonzero for only selected (piezoelectric) dielectrics, the flexoelectric coefficients (components of the fourth order tensor $\mathbf{f}$ ) are, in principle, nonzero for all dielectrics although, of course, they may be negligibly small for many materials. The reader is referred to Tagantsev, ${ }^{9,10}$ who provides an overview of the subject. In a recent work, one of us ${ }^{11}$ has discussed a mathematical framework for flexoelectricity in detail, in addition to providing a review of this subject.

Recently, flexoelectricity has caught the attention of several researchers and, indeed, some have proposed tantalizing notions related to this phenomenon. For instance, Fousek et $a l .{ }^{12}$ were the first to suggest that flexoelectricity should allow fabrication of "piezoelectric composites without using piezoelectric materials." One of us has computationally analyzed such metamaterials, while Cross and co-workers ${ }^{13-15}$ have fabricated nonpiezoelectric tapered pyramidal structures on a substrate that "effectively" act as piezoelectric metamaterials. Flexoelectricity is also seen to play an important role in the characteristics of ferroelectrics, e.g., Catalan et $a l .{ }^{5}$ studied the effect of flexoelectricity on the dielectric constant, polarization, and Curie temperature in ferroelectric thin films under in-plane substrate induced epitaxial strain.

Patently, the strength of the flexoelectric size effects crucially depends on either the numerical values of the flexoelectric coefficients or how large the strain gradients are. The latter is closely linked with the size scale of the structure. Consider two embedded triangular inclusions ${ }^{16}$ (Fig. 1) subject to a stress at two different length scales but with the same aspect ratios. While the strain field remains the same across both length scales, the strain gradients scale as $1 / a_{i}$ (where $a_{i}$ designates a distance between two points inside the inclusion). This simple notion is the essence of the size effect displayed by flexoelectricity.

Flexoelectric coefficients are not readily available, but some reasonable estimates are known for some specific ma- 

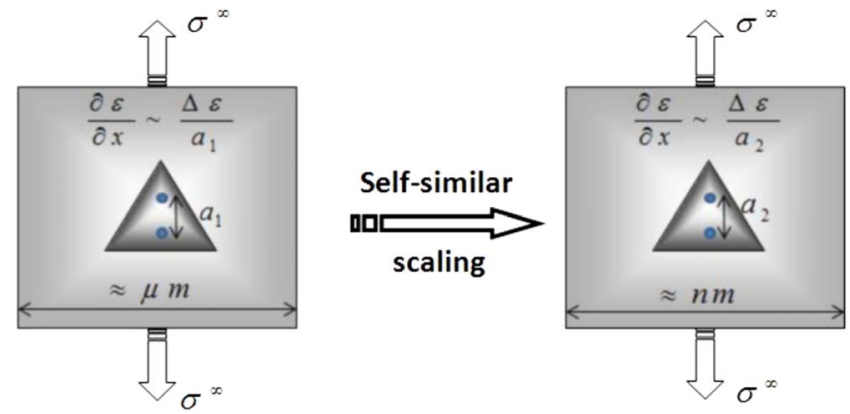

FIG. 1. (Color online) Illustration of size effects due to scaling of strain gradients. Subjected to the same far field stress, two triangular inclusions kept at the same aspect ratio but at different length scales will exhibit strain gradients that scale as $1 / a_{i}$.

terials, e.g., atomistic calculations for graphene (Dumitrica et $a .^{18}$ and Kalinin and Meunier ${ }^{19}$ ) and lattice dynamics for $\mathrm{NaCl}$ (Askar and $\mathrm{Lee}^{20}$ ). Kogan ${ }^{21}$ has argued that for all dielectrics, $e / a\left(\approx 10^{-9} \mathrm{C} / \mathrm{m}\right)$ is an appropriate lower bound for the flexoelectric coefficients, where $e$ is the electronic charge and $a$ is the lattice parameter. Later experiments ( $\mathrm{Ma}$ and $\mathrm{Cross}^{22}$ ) and simple linear chain models of ions (Marvan and Havranek ${ }^{23}$ ) suggested multiplication by relative permittivity for normal dielectrics. Much larger magnitudes $\left(\approx 10^{-6} \mathrm{C} / \mathrm{m}\right)$ of flexoelectric coefficients than this lower bound are observed in certain ceramics. ${ }^{24-26}$ Flexoelectricity, of course, also exists in dielectrics that are already piezoelectric and, in fact, experimental evidence suggests that flexoelectric coefficients are unusually high in such materials see the work of Cross and co-workers ${ }^{13,22,24-26}$ on ferroelectric perovskites such as barium strontium titanate (BST) $\left(f_{\mathrm{BST}}=100 \mu \mathrm{C} / \mathrm{m}\right)$, lead zirconate titanate (PZT) ( $f_{\mathrm{PZT}}=0.5-2 \mu \mathrm{C} / \mathrm{m}$ at lower and higher strain gradients), and lead magnesium niobate $(\mathrm{PMN})\left(f_{\mathrm{PMN}}=4 \mu \mathrm{C} / \mathrm{m}\right)$. Here, we note that the flexoelectric coefficient of ferroelectric materials is quite high even in the paraelectric phase. Quite remarkably, Zubko et al. ${ }^{27}$ have recently published the experimental characterization of the complete flexoelectric tensor for $\mathrm{SrTiO}_{3}$.

In the present work, we analyze the role of flexoelectricity in both piezoelectric and nonpiezoelectric nanostructures. In particular, we focus on the illustrative model problem of a nanoscale cantilever beam to obtain analytical expressions for the "effective" or "apparent" size-dependent piezoelectric coefficient and elastic modulus. The simplicity of the chosen model system allows a facile inference of various physical insights. On this note, we also observe that cantilever beams have important technological ramifications as actuators, sensors, and energy harvester among others. ${ }^{28-33}$ Zhong and Jinhui $^{34}$ used atomic force microscopy to deflect the tips of aligned arrays of piezoelectric cantilever zinc oxide nanowires. Due to bending, such nanoharvesting devices show generated piezoelectric power efficiency up to 30\%. To verify our predictions, we carry out atomistic calculations on both paraelectric and piezoelectric phases of $\mathrm{BaTiO}_{3}$ (BT) nanoscale cantilever beams under bending deformation.

The paper is organized as follows. In Sec. II, we summarize the mathematical framework and the governing equa- tions of flexoelectricity. In Sec. III, we develop solutions for the model nanoscale cantilever beam. Based on the analytical results for this paradigmatic problem, we present the key physical insights in Sec. IV and, in particular, discuss the possibility of giant piezoelectricity at the nanoscale and the size-dependent renormalization of the elastic modulus. In Sec. V, we present our atomistic calculations, and conclude in Sec. VI.

\section{THEORY OF FLEXOELECTRICITY AND GOVERNING EQUATIONS}

In this section, our presentation closely follows Refs. 35-37 including one of our recent papers. ${ }^{11}$ We note that the correct incorporation of flexoelectricity naturally necessitates the inclusion of polarization gradients also (the latter was first introduced by Mindlin ${ }^{35}$ ). The symbol Lin designates the set of all linear transformations, and the associated inner product is defined as $\langle\mathbf{A}, \mathbf{B}\rangle=\operatorname{tr}\left(\mathbf{A}^{T} \mathbf{B}\right)$.

For a dielectric occupying a volume $V$ bounded by a surface $S$ in a vacuum $V^{\prime}$, with a total volume $V^{*}$, Hamilton's principle may be written as

$$
\begin{gathered}
\delta \int_{t_{1}}^{t_{2}} d t \int_{V^{*}}\left(\frac{1}{2} \rho\langle\dot{\mathbf{u}}, \dot{\mathbf{u}}\rangle-H\right) d V+\int_{t_{1}}^{t_{2}} d t\left[\int_{V}(\langle\mathbf{f}, \delta \mathbf{u}\rangle\right. \\
\left.\left.+\left\langle\mathbf{E}^{0}, \delta \mathbf{P}\right\rangle\right) \delta V+\int_{S}\langle\mathbf{t}, \delta \mathbf{u}\rangle d S\right]=0,
\end{gathered}
$$

where $\mathbf{u}, \mathbf{P}, \mathbf{f}, \mathbf{E}^{0}$, and $\mathbf{t}$ are respectively the displacement, the polarization, the external body force, electric field, and surface traction.

The electric enthalpy density $H$ was defined by Toupin ${ }^{38}$ and divided into energy density of deformation and polarization denoted $W^{L}$ and a remainder. By extending the dependence of $W^{L}$ to include both strain and polarization gradients, the electric enthalpy density $H$ takes the following form:

$$
H=W^{L}(\mathbf{S}, \mathbf{P}, \nabla \nabla \mathbf{u}, \nabla \mathbf{P})-\frac{1}{2} \varepsilon_{0}\langle\nabla \varphi, \nabla \varphi\rangle+\langle\nabla \varphi, \mathbf{P}\rangle,
$$

where $\mathbf{S}$ is the symmetric strain tensor, $\varphi$ is the potential of the Maxwell self-field (MS) defined by $\mathbf{E}^{\mathrm{MS}}=-\nabla \varphi$, and $\varepsilon_{0}$ is the permittivity of the vacuum.

Assuming an independent variation of the displacement, polarization, electric potential, and their gradients, the variation of the electric enthalpy density $\delta H$ is

$$
\begin{aligned}
\delta H= & \langle\mathbf{T}, \delta \mathbf{S}\rangle-\langle\overline{\mathbf{E}}, \delta \mathbf{P}\rangle+\langle\widetilde{\mathbf{T}}, \delta \nabla \nabla \mathbf{u}\rangle+\langle\widetilde{\mathbf{E}}, \delta \nabla \mathbf{P}\rangle \\
& -\varepsilon_{0}\langle\nabla \varphi, \delta \nabla \varphi\rangle+\langle\nabla \varphi, \delta \mathbf{P}\rangle+\langle\mathbf{P}, \delta \nabla \varphi\rangle,
\end{aligned}
$$

where

$$
\mathbf{T}=\frac{\partial W^{L}}{\partial \mathbf{S}}, \quad \overline{\mathbf{E}}=-\frac{\partial W^{L}}{\partial \mathbf{P}}, \quad \widetilde{\mathbf{T}}=\frac{\partial W^{L}}{\partial \nabla \nabla \mathbf{u}}, \quad \widetilde{\mathbf{E}}=\frac{\partial W^{L}}{\partial \nabla \mathbf{P}},
$$

$\mathbf{T}$ is the stress tensor, $\overline{\mathbf{E}}$ is the effective local electric force, and $\widetilde{\mathbf{T}}$ and $\widetilde{\mathbf{E}}$ can be interpreted as higher order stress and local electric force, respectively.

Using the chain rule of differentiation, 


$$
\begin{aligned}
\delta H= & \nabla \cdot(\mathbf{T} \cdot \delta \mathbf{u})-\langle\nabla \cdot \mathbf{T}, \delta \mathbf{u}\rangle+\nabla \cdot(\widetilde{\mathbf{T}} \cdot \delta \nabla \mathbf{u}) \\
& -\langle\nabla \cdot \widetilde{\mathbf{T}}, \delta \nabla \mathbf{u}\rangle-\langle\overline{\mathbf{E}}-\nabla \varphi, \delta \mathbf{P}\rangle+\nabla \cdot(\widetilde{\mathbf{E}} \delta \mathbf{P}) \\
& -\langle\nabla \cdot \widetilde{\mathbf{E}}, \delta \mathbf{P}\rangle+\nabla \cdot\left[\left(-\varepsilon_{0} \nabla \varphi+\mathbf{P}\right) \delta \varphi\right] \\
& -\left(-\varepsilon_{0} \Delta \varphi+\nabla \cdot \mathbf{P}\right) \delta \varphi .
\end{aligned}
$$

The kinetic energy in Eq. (3) is written as

$$
\delta \int_{t_{1}}^{t_{2}} d t \int_{V^{*}} \frac{1}{2} \rho\langle\dot{\mathbf{u}}, \dot{\mathbf{u}}\rangle d V=-\int_{t_{1}}^{t_{2}} d t \int_{V^{*}} \rho\langle\ddot{\mathbf{u}}, \delta \mathbf{u}\rangle d V .
$$

Substituting Eq. (7) into the Hamilton principle Eq. (3) and by using the divergence theorem, we find that

$$
\begin{aligned}
& \int_{t_{1}}^{t_{2}} d t \int_{V^{*}}[\langle(-\rho \ddot{\mathbf{u}}+\nabla \cdot \mathbf{T}-\nabla \cdot(\nabla \cdot \widetilde{\mathbf{T}})+\mathbf{f}), \delta \mathbf{u}\rangle+\langle(\overline{\mathbf{E}}-\nabla \varphi \\
& \left.\left.\left.\quad+\nabla \cdot \widetilde{\mathbf{E}}+\mathbf{E}^{0}\right), \delta \mathbf{P}\right\rangle+\left(-\varepsilon_{0} \Delta \varphi+\nabla \cdot \mathbf{P}\right) \delta \varphi\right] d V \\
& \quad+\int_{t_{1}}^{t_{2}} d t \int_{S}[\langle[(-\mathbf{T}+\nabla \cdot \widetilde{\mathbf{T}}) \cdot \mathbf{n}+\mathbf{t}], \delta \mathbf{u}\rangle-\langle\widetilde{\mathbf{E}} \cdot \mathbf{n}, \delta \mathbf{P}\rangle \\
& \left.\quad-\left\langle-\varepsilon_{0} \nabla \varphi+\mathbf{P}, \mathbf{n}\right\rangle \delta \varphi\right] d S=0 .
\end{aligned}
$$

Hence, the equilibrium equations are

$$
\begin{gathered}
\nabla \cdot \boldsymbol{\sigma}+\mathbf{f}=\rho \ddot{\mathbf{u}} \quad \text { where } \boldsymbol{\sigma}=\mathbf{T}-\nabla \cdot \widetilde{\mathbf{T}} \quad \text { in } V, \\
\overline{\mathbf{E}}+\nabla \cdot \widetilde{\mathbf{E}}-\nabla \varphi+\mathbf{E}^{0}=0 \quad \text { in } V, \\
-\varepsilon_{0} \Delta \varphi+\nabla \cdot \mathbf{P}=0 \quad \text { in } V \text { and } \Delta \varphi=0 \quad \text { in } V^{\prime},
\end{gathered}
$$

whereas the corresponding boundary conditions on $\mathbf{S}$ are

$$
\begin{gathered}
\boldsymbol{\sigma} \cdot \mathbf{n}=\mathbf{t} \quad \text { where } \boldsymbol{\sigma}=\mathbf{T}-\nabla \cdot \widetilde{\mathbf{T}}, \\
\widetilde{\mathbf{E}} \cdot \mathbf{n}=0, \\
\left(-\varepsilon_{0}\|\nabla \varphi\|+\mathbf{P}\right) \cdot \mathbf{n}=0 .
\end{gathered}
$$

$\boldsymbol{\sigma}$ may be considered as the actual physical stress experienced by a material point and differs from the Cauchy stress T. The symbol \|\| denotes the jump across the surface or an interface.

Neglecting the contribution of higher order terms (fifth order tensors and higher), the strain energy density can be expanded as

$$
\begin{aligned}
W^{L}(\mathbf{S}, \mathbf{P}, \nabla \nabla \mathbf{u}, \nabla \mathbf{P})= & \frac{1}{2} \mathbf{P} \cdot \mathbf{a} \cdot \mathbf{P}+\frac{1}{2} \nabla \mathbf{P}: \mathbf{b}: \nabla \mathbf{P}+\frac{1}{2} \mathbf{S}: \mathbf{c}: \mathbf{S} \\
& +\mathbf{S}: \mathbf{e}: \nabla \mathbf{P}+\mathbf{S}: \mathbf{d} \cdot \mathbf{P}+\mathbf{P} \cdot \mathbf{g}: \nabla \mathbf{P} \\
& +\mathbf{P} \cdot \mathbf{f}: \nabla \nabla \mathbf{u} .
\end{aligned}
$$

Finally, according to Eq. (6), the constitutive equations are

$$
\begin{gathered}
\mathbf{T}=\frac{\partial W^{L}}{\partial \mathbf{S}}=\mathbf{c}: \mathbf{S}+\mathbf{e}: \nabla \mathbf{P}+\mathbf{d} \cdot \mathbf{P}, \\
\widetilde{\mathbf{T}}=\frac{\partial W^{L}}{\partial \nabla \nabla \mathbf{u}}=\mathbf{f} \cdot \mathbf{P},
\end{gathered}
$$

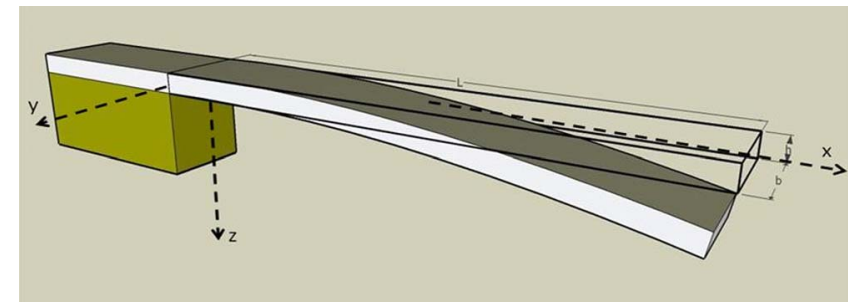

FIG. 2. (Color online) Schematic of a rectangular cantilever beam. Initial and bent configurations are sketched.

$$
\begin{gathered}
-\overline{\mathbf{E}}=\frac{\partial W^{L}}{\partial \mathbf{P}}=\mathbf{a} \cdot \mathbf{P}+\mathbf{g}: \nabla \mathbf{P}+\mathbf{f}: \nabla \nabla \mathbf{u}+\mathbf{d}: \mathbf{S}, \\
\widetilde{\mathbf{E}}=\frac{\partial W^{L}}{\partial \nabla \mathbf{P}}=\mathbf{b}: \nabla \mathbf{P}+\mathbf{e}: \mathbf{S}+\mathbf{g} \cdot \mathbf{P} .
\end{gathered}
$$

The coefficients of the displacement, polarization, and their gradients defined above as $\mathbf{a}, \mathbf{b}, \mathbf{c}, \mathbf{d}, \mathbf{f}, \mathbf{g}$, and $\mathbf{e}$ are material property tensors. The second order tensor $\mathbf{a}$ is the reciprocal dielectric susceptibility. The fourth order tensor $\mathbf{b}$ is the polarization gradient-polarization gradient coupling tensor and $\mathbf{c}$ is the elastic tensor. The fourth order tensor e corresponds to polarization gradient and strain coupling introduced by Mindlin, ${ }^{35}$ whereas $\mathbf{f}$ is the fourth order flexoelectric tensor. $\mathbf{d}$ and $\mathbf{g}$ are the third order piezoelectric tensor and the polarization-polarization gradient coupling tensor, respectively.

\section{MODEL PROBLEM: CANTILEVER NANOBEAM}

Piezoelectric materials generally have symmetry lower than cubic and (even for the latter) analytical calculations are all but impossible for general three-dimensional bodies. A cantilever beam is a model system that degenerates to a onedimensional problem and is, thus, analytically tractable (albeit approximately). Figure 2 depicts the schematic of such a cantilever beam. We note that a closed-form solution of a cantilever predicated on classical piezoelectric theory (excluding the flexoelectric effect) has been derived by Weinberg. ${ }^{39}$ The latter work ignores variation of electric field through the thickness of the beam and, accordingly, is only valid for materials with low electromechanical coupling. Subsequently, Tadmor and $\mathrm{Kosa}^{40}$ have improved upon on that work by taking into account the variation of the electric field in the beam layers.

We adopt the usual assumptions made in analyzing slender beams, e.g., beam thickness is much less than the radius of curvature induced by the mechanical and electrical loading, and that beam cross section is constant along its length. In the adopted $O x y z$ Cartesian coordinate system (Fig. 2), $O x$ corresponds to the centroidal axis of the undeformed beam, $y$ axis is the neutral axis, and the $z$ axis is the symmetry axis. Although a rectangular cross-sectional beam is depicted in Fig. 2, much of the derivation proceeds for an arbitrary cross-sectional shape.

The displacement field is $\mathbf{u}=\mathbf{u}\left(u_{1}(x, z), u_{2}=0, u_{3}(x)\right)$. As is typical in the analysis of beams, the displacement is param- 
etrized with respect to the out-of-plane displacement component:

$$
\begin{gathered}
u_{3}=w(x), \\
u_{1}=-z \frac{d u_{3}(x)}{d x}=-z \frac{d w(x)}{d x}, \\
u_{2}=0 .
\end{gathered}
$$

For narrow beams $(b<5 h)$, it is typical to assume that the stresses $\sigma_{33}=\sigma_{3}=0$ and $\sigma_{22}=\sigma_{2}=0$. The only relevant electric field component is $E_{3}$. According to the physical stress defined in Eq. (10), the nonvanishing component $\sigma_{11}$ is

$$
\sigma_{11}=\sigma_{1}=T_{11}-T_{111,1}-T_{113,3} .
$$

Without loss of generality, we now assume tetragonal $4 \mathrm{~mm}$ material symmetry. Most piezoelectrics are of the latter or higher symmetry (e.g., PZT 5H). Accordingly, we can rewrite Eq. (15) as

$$
\sigma_{1}=c_{1} S_{1}+\left(e_{13}-f_{13}\right) P_{3,3}+d_{31} P_{3},
$$

in which the Voigt notation is used for the different coefficients and $c_{1}, d_{31}, e_{13}$, and $f_{13}$ designate respectively the elastic modulus, the piezoelectric constant, the polarization gradient and strain coupling constant, and the flexoelectric coefficient of the one-dimensional beam.

$S_{1}$ is the axial strain which can be explicitly written under the beam assumptions as function of the radius curvature $R(x)$ :

$$
S_{1}(x, z)=-\frac{z}{R(x)}=-z \frac{d^{2} w(x)}{d x^{2}} .
$$

Equation (14) may be rewritten with a somewhat simpler notation as

$$
\sigma_{1}=Y S_{1}+(e-f) P_{3,3}-Y d P_{3} .
$$

Here, $Y=c_{1}, e=e_{13}, f=f_{13}$, and $d=-d_{31} / Y$.

The notation in Eq. (18) facilitates subsequent comparison with results obtained by Tadmor and Kosa ${ }^{40}$ for classical piezoelectric beams.

Finally, the electric field induced by the polarization due to piezoelectricity and flexoelectricity (strain gradient term) is expressed as

$$
E_{3}=\varepsilon_{0}^{-1} \chi_{33}^{-1} P_{3}-f_{55} S_{11,3},
$$

where $\chi_{33}^{-1}=\varepsilon_{0} a_{33}=\chi^{-1}$ is the reciprocal dielectric susceptibility. by

The total electric displacement in the $z$ direction is given

$$
D_{3}=d \sigma_{1}+\varepsilon E_{3}+f_{55} S_{11,3},
$$

with $\varepsilon=\varepsilon_{33}$ the dielectric constant. Predicated on onedimensional (1D) beam assumptions, we have from Eq. (20)

$$
E(x, z)=\frac{1}{\varepsilon}\left[D_{3}(x)-d \sigma_{1}(x, z)-f_{55} S_{11,3}\right] .
$$

We define the "through-layer" average of any quantity as

$$
\bar{T}(x)=\frac{1}{h} \int_{\text {Layer }} T(x, z) d z .
$$

Since the applied voltage difference is constant along the beam,

$$
V=-\frac{D_{3}(x) h}{\varepsilon}+\frac{d h}{\varepsilon} \bar{\sigma}_{1}+\frac{f_{55} h}{\varepsilon} \bar{S}_{11,3} .
$$

We may, thus, write

$$
D_{3}(x)=-\frac{\varepsilon V}{h}+d \bar{\sigma}_{1}+f_{55} \bar{S}_{11,3} .
$$

From Eq. (18), the average layer stress is then

$$
\bar{\sigma}_{1}=(e-f) \bar{P}_{3,3}-Y d \bar{P}_{3} .
$$

Assuming a linear variation of the electric field in $z$ and that the average layer electric field and voltage are respectively equal to $-V / h$ and $V$, we find that

$$
\bar{E}_{3}=-\frac{V}{h}, \quad \bar{E}_{3,3}=-\frac{24 V}{h^{2}}
$$

and, hence, $\bar{P}_{3}$ and $\bar{P}_{3,3}$.

Substituting Eq. (24) into Eq. (21) with Eq. (25), we obtain an equation to solve for the electric polarization

$$
-\frac{V \varepsilon_{0} \chi}{h}-\frac{d}{\varepsilon}\left[\sigma_{1}(x, z)-\bar{\sigma}_{1}\right]=P(x, z)-f^{\prime} S_{11,3}
$$

in which $f^{\prime}=f_{55}$.

Solving Eq. (26), we obtain

$$
\begin{aligned}
P(x, z)= & -\underbrace{\frac{V \varepsilon_{0} \chi}{h}}_{\text {electrostatic }}+\underbrace{\frac{\xi}{d R(x)}}_{\text {pure piezoelectricity }}-\underbrace{\frac{f^{\prime}}{R(x)}}_{\text {pure flexoelectricity }} \\
& -\underbrace{\frac{\xi^{2}(e-f)}{d^{2} Y R(x)}-\frac{24 V \varepsilon_{0} \chi \xi(e-f)}{d Y h^{2}}}_{\text {piezoelectricity-flexoelectricity interaction }},
\end{aligned}
$$

where $\xi=k_{e}^{2}=k^{2} /\left(1-k^{2}\right)$ is defined as the square of the expedient coupling coefficient ${ }^{41} k_{e}$, and $k=\sqrt{Y d^{2} / \varepsilon}$ is the socalled electromechanical coupling (EMC) coefficient.

The first term in Eq. (27) corresponds to polarization due to an applied voltage; the second is due to a pure piezoelectric effect; the third term is due to a pure strain gradient or flexoelectric effect (polarization exists even in the absence of applied voltage and piezoelectric effect as long as the strain is nonuniform), whereas the last two terms correspond to combined piezoelectric and flexoelectric contributions and, thus, informs us of the nonlinear interaction between flexoelectricity and piezoelectricity.

Note that our solution coincides with the results of Tadmor and $\operatorname{Kosa}^{40}$ if we neglect the higher order contribution of polarization and strain gradients $\left(e \rightarrow 0\right.$ and $\left.f, f^{\prime} \rightarrow 0\right)$. In addition, if we further disregard the EMC $(\xi \rightarrow 0)$, we recover the classical result for a simple dielectric in which the electric field is a constant $-V / h$. 
To proceed further, it is expedient to define the strain energy $U$ :

$$
U=\frac{1}{2} \iint_{V} \int T_{i j} S_{i j} d V+\frac{1}{2} \iint_{V} \int T_{i j m} u_{i, j m} d V .
$$

For the case of the $1 \mathrm{D}$ beam, it reduces to

$$
U=-\frac{1}{2} \int_{x=0}^{L} \hat{M}(x) \frac{d^{2} w(x)}{d x^{2}} d x-\frac{1}{2} \int_{x=0}^{L} \hat{P}(x) \frac{d^{2} w(x)}{d x^{2}} d x
$$

where

$\hat{M}(x)=\iint_{A} z T_{1}(x, z) d y d z$ and $\hat{P}(x)=\iint_{A} f P_{3}(x, z) d y d z$

are the resultant moment and the higher order resultant moment, respectively.

In the absence of body forces, the work done by external forces due only to transverse loading $q(x)$ is

$$
W(x)=\int_{x=0}^{L} q(x) w(x) d x .
$$

The total potential energy $\Pi$ is obtained from Eqs. (29) and (31) as

$$
\begin{aligned}
\prod= & U-W=-\frac{1}{2} \int_{x=0}^{L}[\hat{M}(x)+\hat{P}(x)] \frac{d^{2} w(x)}{d x^{2}} d x \\
& -\int_{x=0}^{L} q(x) w(x) d x .
\end{aligned}
$$

Its first variation is derived in a similar form as given by Ref. 42

$$
\begin{aligned}
\delta \prod= & {\left[-(\hat{M}(x)+\hat{P}(x)) \delta w^{\prime}(x)\right]_{0}^{L} } \\
& +\left[\left(\frac{d \hat{M}(x)}{d x}+\frac{d \hat{P}(x)}{d x}\right) \delta w(x)\right]_{0}^{L} \\
& -\int_{0}^{L}\left(\frac{d^{2} \hat{M}(x)}{d x^{2}}+\frac{d^{2} \hat{P}(x)}{d x^{2}}+q(x)\right) \delta w(x) d x .
\end{aligned}
$$

By using the principle of minimum potential energy $(\delta \Pi$ $=0$, e.g., Ref. 43) and the fundamental lemma of calculus of variation (e.g., Ref. 44), we have the following governing equation from Eq. (33):

$$
\frac{d^{2} \hat{M}(x)}{d x^{2}}+\frac{d^{2} \hat{P}(x)}{d x^{2}}+q(x)=0, \quad \forall x \in(0, L) .
$$

The corresponding boundary conditions prescribed at the beam ends $(x=0$ and $x=L)$ are

$$
\hat{M}(x)+\hat{P}(x) \quad \text { or } \quad \frac{d w(x)}{d x},
$$

$$
\frac{d(\hat{M}(x)+\hat{P}(x))}{d x} \text { or } \quad w(x) .
$$

From Eqs. (30), (13), and (28), we can show that

$$
\hat{M}(x)=-Y I(1+\xi) \frac{d^{2} w(x)}{d x^{2}},
$$

$$
\hat{P}(x)=-A f\left[\frac{V \varepsilon_{0} \chi}{h}+\frac{f^{\prime}}{R(x)}+\frac{\xi^{2}(e-f)}{d^{2} Y R(x)}+\frac{24 V \varepsilon_{0} \chi \xi(e-f)}{d Y h^{2}}\right],
$$

where $I=\iint_{A} z^{2} d A$ is the second moment of cross-sectional area $A$.

Thus, the equilibrium Eq. (34) becomes

$$
G \frac{d^{4} w(x)}{d x^{4}}=q(x)
$$

where $G$ is the beam bending rigidity defined as

$$
G=Y I\left[1+\xi+\frac{A f f^{\prime}}{Y I}+\frac{A f \xi^{2}(e-f)}{d^{2} Y^{2} I}\right] .
$$

Once again, we point out that if we ignore the polarization and strain gradient effects $\left(e \rightarrow 0\right.$ and $\left.f, f^{\prime} \rightarrow 0\right)$, we recover the same bending rigidity as in Ref. 40 Also, if we neglect the EMC $(\xi \rightarrow 0)$, we recover the classical bending rigidity for a beam $G=Y I$. Note that in the absence of piezoelectricity $(\xi \rightarrow 0)$, the renormalized bending rigidity is $G=Y I$ $+A f f^{\prime}$ due to flexoelectric effect.

The preceding derivation is for an arbitrary crosssectional beam. As a concrete example, consider a rectangular cantilever beam subjected to a transversal point load $N$. The corresponding boundary conditions from Eq. (35) are

$$
\begin{gathered}
w(0)=0 \quad \text { and }\left.\quad \frac{d w(x)}{d x}\right|_{x=0}=0, \\
\hat{M}(L)+\hat{P}(L)=0 \quad \text { and }\left.\quad \frac{d(\hat{M}(x)+\hat{P}(x))}{d x}\right|_{x=L}=N .
\end{gathered}
$$

In the absence of distributed transverse loading $[q(x)=0]$, the homogeneous equilibrium equation becomes

$$
G \frac{d^{4} w(x)}{d x^{4}}=0,
$$

where the solution is in the form

$$
G w(x)=\frac{a_{1}}{6} x^{3}+\frac{a_{2}}{2} x^{2}+a_{3} x+a_{4} .
$$

By means of the boundary conditions in Eq. (39), the beam deflection is then

$$
w(x)=\frac{N x^{2}(3 L-x)}{6 G}
$$

in which the bending rigidity $G$ is defined by Eq. (38) with $I=b h^{3} / 12$ and $A=b h$. 


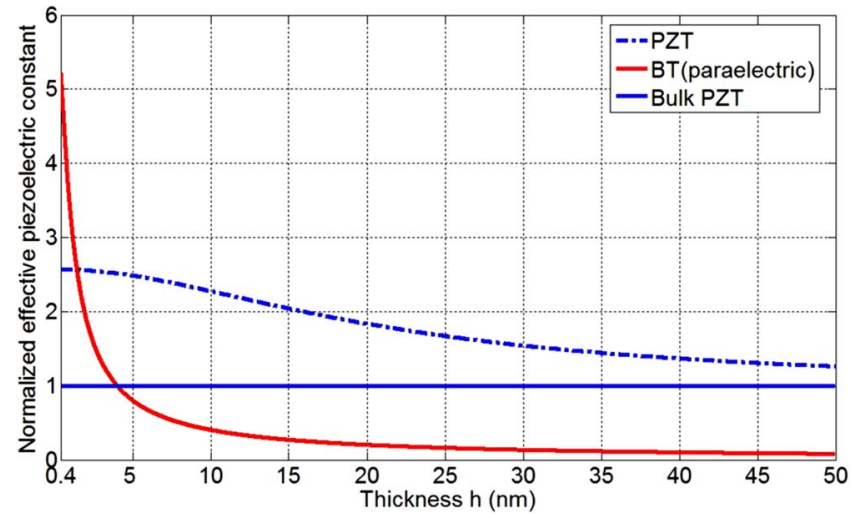

FIG. 3. (Color online) Normalized effective piezoelectric constant of deformed PZT (dashed blue; dashed dark gray in print) and nonpiezoelectric BT beams (solid red; light gray in print). The normalization is done with respect to the bulk piezoelectric constant of PZT (solid blue; dark gray in print) $\left(d_{P Z T}=-274 \mathrm{pC} / \mathrm{N}\right)$ and piezoelectric phase of BT $\left(d_{B T}=-78 \mathrm{pC} / \mathrm{N}\right)$.

Thus, we may use the classical well-known beam equation for deflection provided the rigidity (or, in effect, the elastic modulus) is renormalized according to Eq. (38).

\section{PHYSICAL INSIGHTS, POSSIBILITY OF "GIANT" SIZE-DEPENDENT PIEZOELECTRICITY, AND SCALING OF ELASTIC MODULUS}

Based on the derivation in the preceding section, we may define an effective or "renormalized" piezoelectric constant which has contributions from both classical piezoelectricity and flexoelectricity.

From Eq. (38), we define the effective coupling coefficient as

$$
\xi_{\text {eff }}=\xi+\frac{12 f(e-f) \xi^{2}}{Y^{2} d^{2} h^{2}}+\frac{12 f f^{\prime}}{Y h^{2}} .
$$

Consequently, the effective piezoelectric coefficient is

$$
d_{e f f}=\sqrt{\frac{\varepsilon}{Y} \frac{\xi_{e f f}}{\left(1+\xi_{e f f}\right)}} .
$$

Figure 3 shows that for piezoelectric PZT cantilevers (dashed line), the effective piezoelectric constant is increased by $75 \%$ of the PZT bulk value $\left(d_{P Z T}=-274 \mathrm{pC} / \mathrm{N}\right)$ at $20 \mathrm{~nm}$. Even though cubic BT is not piezoelectric (red solid line), we still see a large apparent piezoelectric response below $10 \mathrm{~nm}$. At $8 \mathrm{~nm}$, the apparent piezoelectric response of BT is $50 \%$ that of the bulk BT piezoelectric constant $(d B T$ $=-78 \mathrm{pC} / \mathrm{N})$. At $2 \mathrm{~nm}$, the apparent piezoelectric response is double the one generated by a piezoelectric BT beam. An extremely high apparent piezoelectric response is seen at smaller sizes, reaching almost five times the piezoelectric BT constant.

$\mathrm{Ma}$ and Cross report that ferroelectric phase (piezoelectric) $\mathrm{BT}$ has a high flexoelectric constant estimated ${ }^{45}$ to be $f_{B T}=50 \mu \mathrm{C} / \mathrm{m}$. Figure 4 shows that for piezoelectric BT, the effective piezoelectric response increases by $20 \%$ of its bulk

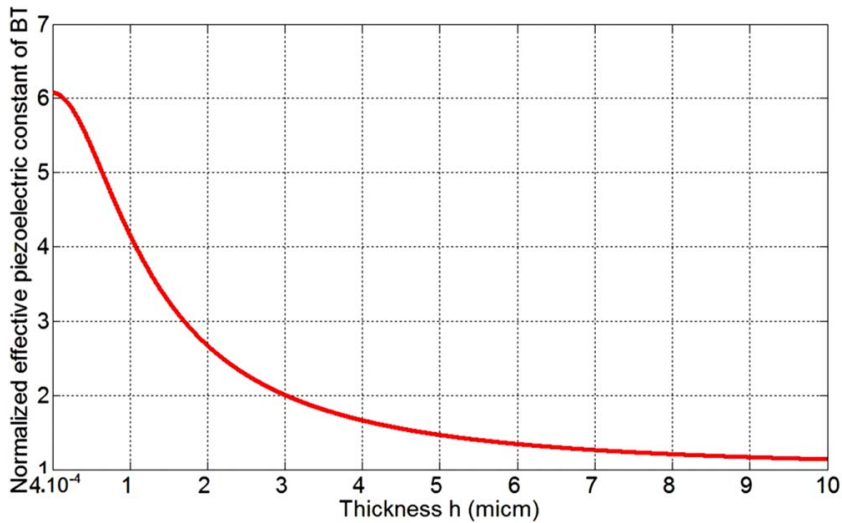

FIG. 4. (Color online) Normalized effective piezoelectric constant of tetragonal (piezoelectric) BT beam. An enhancement of $20 \%$ of its bulk value at $8 \mu \mathrm{m}$ and a $500 \%$ increase at $5 \mathrm{~nm}$ are observed.

value at $8 \mu \mathrm{m}$ and exhibits a giant $500 \%$ increase at $5 \mathrm{~nm}$.

Our theoretical results indicate that the apparent piezoelectric response is determined by a synergistic addition between piezoelectricity and flexoelectricity [e.g., Eq. (27)]. By comparing PZT and piezoelectric BT results, the noteworthy increase in the piezoelectric response occurs at vastly different length scales. The effective piezoelectric constant as defined previously in Eq. (44) depends on both piezoelectric constant (EMC) and the flexoelectric constant. The piezoelectric constant for PZT is higher than BT but it is of the same order of magnitude. However, the flexoelectric constant of BT is 2 orders of magnitude higher than that of PZT $\left(f_{B T}=100 f_{P Z T}\right)$, which explains the difference in the length scales at which the enhancement is observed. In the case of nonpiezoelectric (paraelectric) BT, the only contribution to the effective piezoelectric response is due to flexoelectricity. Therefore, the effect is smaller and only occurs at small scales (tens of nanometers).

We now show that flexoelectricity also impacts the observed or apparent elastic modulus. The normalized effective Young's modulus (with bulk value) is defined as [see Eq. (38) also]

$$
Y^{\prime}=\frac{G}{Y I}=\left[1+\xi+\frac{12 f(e-f) \xi^{2}}{Y^{2} d^{2} h^{2}}+\frac{12 f f^{\prime}}{Y h^{2}}\right] .
$$

To illustrate our results, we pick the following values for the different parameters: A PZT $5 H$ beam with rectangular cross section defined by $b=2 h(b<5 h$, plane stress) and $L=20 h$, loaded with a force magnitude $N=100 \mu \mathrm{N}$ so that we remain in the elastic domain. The flexoelectric coefficient $f$ is obtained from Ref. $12, f_{P Z T}=0.5 \mu \mathrm{C} / \mathrm{m}$. A $1 / h^{2}$ scaling is evident in Eq. (45) and is illustrated in Fig. 5: smaller beams appear stiffer due to the flexoelectric effect (dashed line). Note that the normalized effective Young's modulus according to Tadmor and Kosa ${ }^{40}$ is a little larger than 1 because of the EMC contribution.

\section{ATOMISTIC SIMULATIONS}

In previous sections, based on the phenomenon of flexoelectricity and an appropriate mathematical description, we 


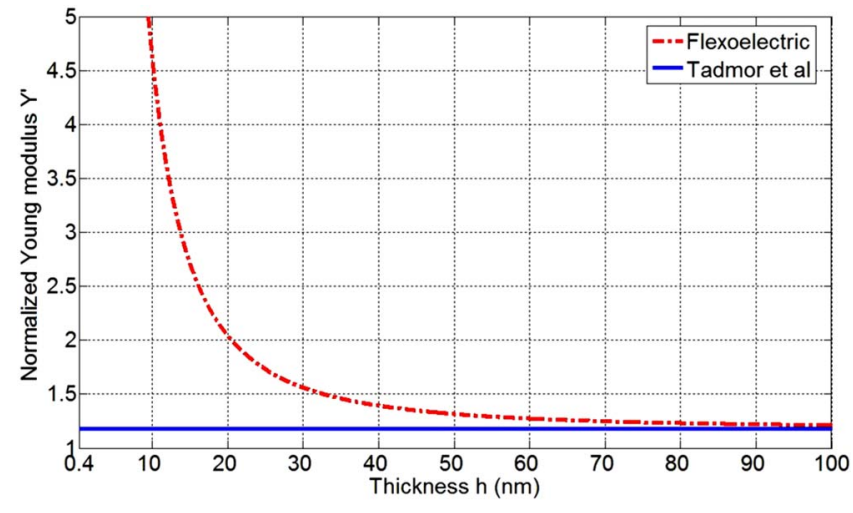

FIG. 5. (Color online) Normalized Young's modulus of a rectangular PZT cantilever beam. The dashed line illustrates the size dependency of the elastic modulus and exhibits a $1 / h^{2}$ scaling where $h$ is the beam thickness. The horizontal solid line is for the results of Tadmor and Kosa, for a classical piezoelectric beam that excludes the flexoelectric effect.

have argued the possibility of giant piezoelectricity in piezoelectric nanostructures and certainly an enhancement even in nonpiezoelectric ones. In this section, we present discrete atomistic calculations based on a (quantum mechanically derived) force field to confirm some of our predictions. We have avoided atomistic calculation of PZT since the coreshell potential available for it is not parametrized appropriately for the physical insights sought by the present work. Therefore, we focused our attention mainly on BT. At temperature above the Curie temperature $T_{C}$ of $393 \mathrm{~K}$, BT is in its stable paraelectric cubic phase $(P m 3 m)$. Below $T_{C}$, BT undergoes three ferroelectric phase transitions. The cubic structure changes to tetragonal $(P 4 \mathrm{~mm})$ symmetry at $T_{C}$, orthorhombic $(A m m 2)$ at $278 \mathrm{~K}$, and the last phase transition, rhombohedral $(R 3 m)$, occurs at $183 \mathrm{~K}$. In prior work, ${ }^{46}$ one of us has developed a suitable polarizable charge distribution force field for BT to use in molecular dynamics (MD) simulations based on $a b$ initio quantum mechanical calculations. One distinctive feature of this force field is that charge transfer and atomic polarization are treated self-consistently and is, thus, quite appropriate for studying ferroelectrics. The charge is described as a Gaussian distribution for each of the core and the shell. The total core charge has positive fixed amplitude centered on the nucleus, whereas the negative valence (shell) charge is determined via charge equilibration and is allowed to move off the nuclear center. The two Gaussian charge distributions interact with Coulombic (electrostatic) forces. Nonbonded interactions between neutral atoms and molecules (short range Pauli repulsion and long range attractive van der Waals dispersion) are described by the Morse potential. Our previous MD calculations ${ }^{46}$ indicate that the polarizable charge distribution force field potential for BT is able to correctly predict experimentally observed paraelectric (cubic) to ferroelectric (tetragonal) phase transition among other features. One of us has also recently successfully used it to study antiferroelectricity in cubic and ferroelectric phases of BT. ${ }^{47}$

Our calculations were carried out using MST package. ${ }^{48}$ Beam thicknesses were varied from 1 single unit cell $(4.01 \AA$

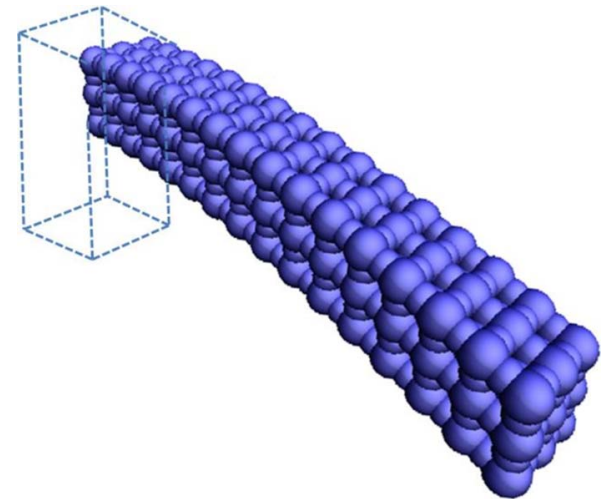

FIG. 6. (Color online) Atomistic representation of a cantilever beam under bending. The square dotted block is for aesthetic perspective.

as lattice parameter) to $2 \mathrm{~nm}$, while length was set to $4 \mathrm{~nm}$. Several simulations were performed and results were averaged over all the runs. To reproduce our theoretical work conditions, simulated rectangular cantilevers were held fixed at one side then bent to the shape dictated by the simple 1D deflection solution defined previously in Eq. (40) (Fig. 6-deflection amplified for clarity).

For a given strain gradient, we determine the average polarization for different runs with different BT beam sizes in both ferroelectric and paraelectric phases. In the case of nonpiezoelectric BT (Fig. 7), MD calculations are in good agreement with the predictions of our theoretical model from the previous section. Only a few points calculated by MD are shown, and the solid line is interpolated using the least square technique, providing a guide for the eye.

For piezoelectric BT, as shown in the previous section, the effective piezoelectric response shows an enhancement at a higher length scale of a few micrometers and reaches gigantic proportions at the nanoscale (Fig. 8). Such giant enhancements are duly confirmed by atomistic calculations.

There are, of course, some (inconsequential) differences between the theoretical model and MD results. The theory is

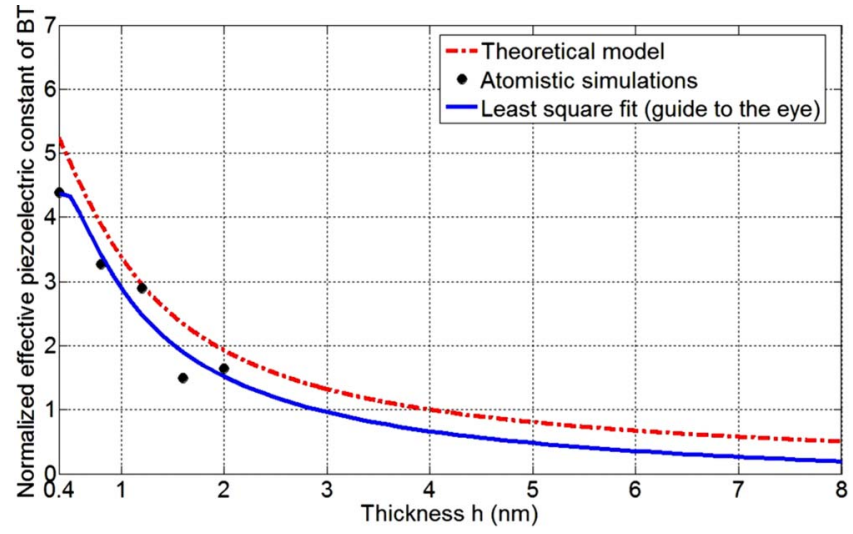

FIG. 7. (Color online) Normalized effective piezoelectric constant of cubic (nonpiezoelectric) BT. Only a few points are obtained from atomistic simulations. The least square fit shows good agreement with the predictions of the theoretical model. 


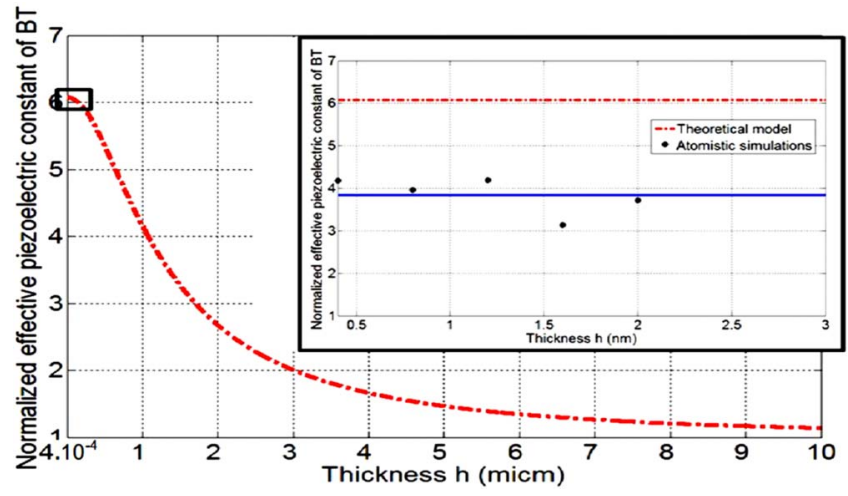

FIG. 8. (Color online) Normalized effective piezoelectric constant of tetragonal (piezoelectric) BT. Since the atomistic calculations were carried for very small sizes, the right inset corresponds to a zoomed-in view around $3 \mathrm{~nm}$. The atomistic results fluctuate around a constant value [least square fit (solid line)] and qualitatively match the theoretical predictions.

developed under the assumptions of a simplified 1D problem, whereas the simulations are carried out on threedimensional nanostructures. In addition, our model sensitively depends on several material properties (Young's modulus, dielectric, and piezoelectric and flexoelectric constants), the values of which could be over- or underestimated by the experimental values we have used. At such small scales, other phenomena, in particular, surface piezoelectricity and/or flexoelectricity, ${ }^{9}$ which are not taken into account by our model, may become important.

We have also computed and contrasted the effective elastic modulus with our theoretical results. The energy difference between the bent beam configuration and the undeformed one is the strain energy or the work done by the applied force. The strain energy $U$ is

$$
U=\frac{1}{2} \int_{V} \sigma S d v=\frac{1}{2} Y I \int_{0}^{L} \frac{1}{R^{2}(x)} d x .
$$

We estimate the normalized effective Young's modulus from the following relation:

$$
Y^{\prime}=\sqrt{\frac{N^{2} L^{3}}{6 Y I U}} .
$$

We note that a similar technique was used by Miller and Shenoy ${ }^{49}$ to explain atomistically the size dependency of Young's modulus of nanosized elements and the flexural rigidity of beams in bending due to surface energy effects.

The atomistic results for Young's modulus for BT are contrasted with theoretical ones in Fig. 9. Once again, down to about $2 \mathrm{~nm}$ or so, there is good agreement (and below which, as already explained, results diverge).

\section{SUMMARY}

We have argued that flexoelectricity exhibits a size effect and, thus, should have important ramifications for the apparent piezoelectric and elastic behavior of nanostructures. Cer-

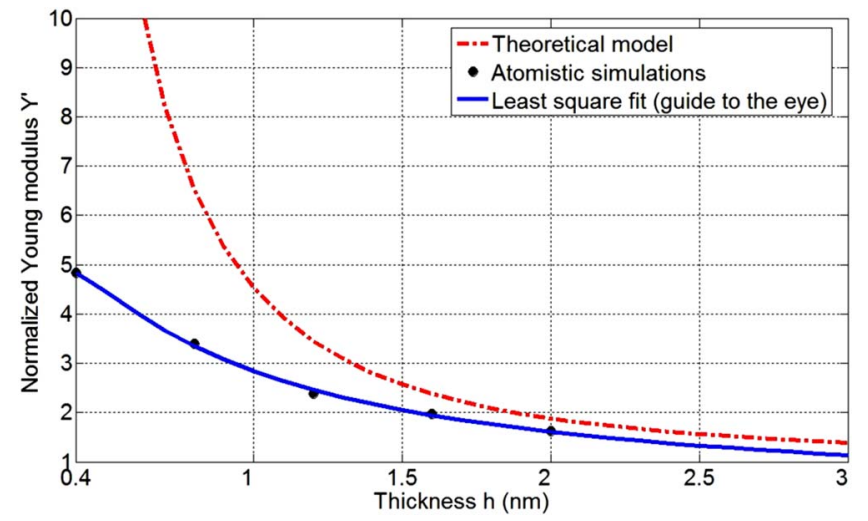

FIG. 9. (Color online) Normalized Young's modulus for a BT cantilever beam. The least square fit of the atomistic simulations demonstrates reasonable agreement down to $2 \mathrm{~nm}$.

tainly in some dielectrics where flexoelectric coefficients are quite high and coupled with large strain gradients possible at the nanoscale, the effect of flexoelectricity can be nontrivial. In particular, using a model system of a cantilever nanobeam, we are able to analytically show that in materials that are already piezoelectric, the effect of flexoelectricity is multiplicative and combines nonlinearly with the intrinsic piezoelectricity. The nonlinear flexoelectric-piezoelectric interaction manifests itself as a giant increase in the apparent piezoelectric response at small sizes for materials that are intrinsically piezoelectric (duly confirmed via accurate atomistic calculations for BT). As is well known in the classical piezoelectricity literature, a polarized elastic solid shows a renormalized (size independent) elastic constant. This is true in flexoelectricity induced elasticity renormalization as well, although the behavior is size dependent and scales as $\sim 1 / h^{2}$.

We find it interesting that classical piezoelectric theory when supplemented with flexoelectricity is able to capture the electromechanical behavior of nanostructures almost down to $2 \mathrm{~nm}$. Needless to say, without incorporation of flexoelectricity, the size effects observed in the atomistic calculations cannot be reconciled. An auxiliary benefit of the present work, thus, is that continuum piezoelectricity duly supplemented with flexoelectricity may be employed to study nanoscale piezoelectricity in a computationally expedient manner rather than using atomistic calculations which have clear computational limits in terms of system size and computational expense.

Currently, very little experimental work is available on piezoelectricity bent nanobeam as it is highly challenging to perform controlled experiments at that scale. In that regard, we note that in some cases (e.g., in piezoelectric phase of $\mathrm{BaTiO}_{3}$ ), the size effect predicted by us are also manifest at micron size beams, thus providing a facile route for experimental verification of our presented scaling laws. Furthermore, the approach and conclusions of this work will remain relevant for same order of magnitude structures such as lattice-mismatched epitaxial thin films. ${ }^{50}$ The latter work examined the influence of strain gradients (through flexoelectric coupling) on the ferroelectric properties of films with decreasing thickness. Another example is the case of asym- 
metric three-component ferroelectric superlattices,${ }^{51}$ where authors confirm enhancement in polarization by similar phenomena (breaking the inversion symmetry of the lattice).

Our theoretical model neglects some effects that may become important at small sizes, e.g., surface flexoelectricity and surface piezoelectricity. ${ }^{9}$ Regarding the latter, we have minimized its influence in atomistic calculations by ensuring the centrosymmetry of surfaces. Surface flexoelectricity has been discussed at length by Tagantsev ${ }^{9}$ and is not included in our theoretical model (although this phenomenon is automatically accounted for in the atomistic calculations). Evidently, surface flexoelectricity is likely to be important only below $2 \mathrm{~nm}$ or so for the materials we have investigated (given the close agreement up to that point between our atomistic and theoretical results).

\section{ACKNOWLEDGMENTS}

M.S.M. acknowledges useful discussions with Takahiro Shimada on PZT core-shell potential. The authors are also grateful to Gillian C. Lynch for use of CERIUS2 software to handle some aspects of the calculations. P.S. and M.S.M. acknowledge financial support from NSF NIRT Grant No. CMMI 0708096 and Texas ARP.
*Corresponding author; psharma@uh.edu

${ }^{1}$ We ignore here the possibility of the nonlinear but universal coupling due to electrostriction (see Ref. 2 for a detailed discussion of nonlinear electromechanical effects). Electrostriction becomes operative at very high electric fields. In addition, an inverse electrostriction effect does not exist, i.e., deformation does not produce an electric field.

${ }^{2}$ G. A. Maugin, Continuum Mechanics of Electromagnetic Solids (North-Holland, Amsterdam, 1988).

${ }^{3}$ J. F. Nye, Physical Properties of Crystals: Their Representation by Tensors and Matrices (Oxford University Press, Oxford, 2004).

${ }^{4}$ E. V. Bursian and N. N. Trunov, Fiz. Tverd. Tela (Leningrad) 16, 1187 (1974).

${ }^{5}$ G. Catalan, L. J. Sinnamon, and J. M. Gregg, J. Phys.: Condens. Matter 16, 2253 (2004).

${ }^{6}$ V. L. Indenbom, E. B. Loginov, and M. A. Osipov, Kristallografiya 26, 1157 (1981).

${ }^{7}$ R. B. Meyer, Phys. Rev. Lett. 22, 918 (1969).

${ }^{8}$ D. Schmidt, M. Schadt, and W. Helfrich, Z. Naturforsch. A 27A, 277 (1972).

${ }^{9}$ A. K. Tagantsev, Phys. Rev. B 34, 5883 (1986).

${ }^{10}$ A. K. Tagantsev, Phase Transitions 35, 119 (1991).

${ }^{11}$ R. Maranganti, N. D. Sharma, and P. Sharma, Phys. Rev. B 74, 014110 (2006).

${ }^{12}$ J. Fousek, L. E. Cross, and D. B. Litvin, Mater. Lett. 39, 287 (1999).

${ }^{13}$ L. E. Cross, J. Mater. Sci. 41, 53 (2006).

${ }^{14}$ J. Y. Fu, W. Zhu, N. Li, and L. E. Cross, J. Appl. Phys. 100, 024112 (2006).

${ }^{15}$ M. Wenhui and L. E. Cross, Appl. Phys. Lett. 88, 232902 (2006).

${ }^{16}$ We consider a noncentrosymmetric inclusion; otherwise, the average polarization would be zero. See our recent paper on the discussion of such symmetry effects (Ref. 17).

${ }^{17}$ N. D. Sharma, R. Maranganti, and P. Sharma, J. Mech. Phys. Solids 55, 2238 (2007).

${ }^{18}$ T. Dumitrica, C. M. Landis, and B. I. Yakobson, Chem. Phys. Lett. 360, 182 (2002).

${ }^{19}$ S. V. Kalinin and V. Meunier, Phys. Rev. B 77, 033403 (2008).

${ }^{20}$ A. Askar and P. C. Y. Lee, Phys. Rev. B 9, 5291 (1974).

${ }^{21}$ S. M. Kogan, Fiz. Tverd. Tela (Leningrad) 5, 2829 (1963).

${ }^{22}$ W. Ma and L. E. Cross, Appl. Phys. Lett. 78, 2920 (2001).

${ }^{23}$ M. Marvan and A. Havranek, Solid State Commun. 101, 493 (1997).
${ }^{24}$ W. Ma and L. E. Cross, Appl. Phys. Lett. 79, 4420 (2001).

${ }^{25}$ W. Ma and L. E. Cross, Appl. Phys. Lett. 81, 3440 (2002).

${ }^{26}$ W. Ma and L. E. Cross, Appl. Phys. Lett. 82, 3293 (2003).

${ }^{27}$ P. Zubko, G. Catalan, P. R. L. Welche, A. Buckley, and J. F. Scott, Phys. Rev. Lett. 99, 167601 (2007).

${ }^{28}$ A. Gupta, D. Akin, and R. Bashir, Appl. Phys. Lett. 84, 1976 (2004).

${ }^{29}$ T. J. Huang, B. Brough, C. M. Hoa, Y. Liu, A. H. Flood, P. A. Bonvallet, H. R. Tseng, J. F. Stoddart, M. Baller, and S. Magonov, Appl. Phys. Lett. 85, 5391 (2004).

${ }^{30}$ L. Mateu and F. Moll, J. Intell. Mater. Syst. Struct. 16, 835 (2005).

${ }^{31}$ P. Muralt, J. Micromech. Microeng. 10, 136 (2000).

${ }^{32}$ R. S. Pereira, Biochem. Pharmacol. 62, 975 (2001).

${ }^{33}$ S. Roundy, P. K. Wright, and J. Rabaey, Energy Scavenging for Wireless Sensor Networks with Special Focus on Vibrations (Kluwer Academic, Boston, 2004).

${ }^{34}$ L. W. Zhong and S. Jinhui, Science 312, 242 (2006).

${ }^{35}$ R. D. Mindlin, Int. J. Solids Struct. 4, 637 (1968).

${ }^{36}$ J. P. Nowacki and R. K. T. Hsieh, Int. J. Eng. Sci. 24, 1655 (1986).

${ }^{37}$ E. Sahin and S. Dost, Int. J. Eng. Sci. 26, 1231 (1988).

${ }^{38}$ R. A. Toupin, Arch. Ration. Mech. Anal. 11, 385 (1962).

${ }^{39}$ M. S. Weinberg, J. Microelectromech. Syst. 8, 529 (1999).

${ }^{40}$ E. B. Tadmor and G. Kosa, J. Microelectromech. Syst. 12, 899 (2006)

${ }^{41}$ T. Ikeda, Fundamentals of Piezoelectricity (Oxford University Press, Oxford, 1996).

${ }^{42}$ S. K. Park and X.-L. Gao, J. Micromech. Microeng. 16, 2355 (2006).

${ }^{43}$ D. J. Steigmann, IMA J. Appl. Math. 48, 195 (1992).

${ }^{44}$ X. L. Gao and S. Mall, Int. J. Solids Struct. 38, 855 (2001).

${ }^{45}$ W. Ma and L. E. Cross, Appl. Phys. Lett. 88, 232902 (2006).

${ }^{46}$ W. A. Goddard III, Q. Zhang, M. Uludogan, A. Strachan, and T. Cagin, in Fundamental Physics of Ferroelectrics, edited by R. E. Cohen (American Institute of Physics, Melville, NY, 2002), p. 45.

${ }^{47}$ Q. Zhang, T. Cagin, and W. A. Goddard III, Proc. Natl. Acad. Sci. U.S.A. 103, 14695 (2006).

${ }^{48}$ Q. Zhang, Ph.D. thesis, California Institute of Technology, 2004.

${ }^{49}$ R. E. Miller and V. B. Shenoy, Nanotechnology 11, 113 (2000).

${ }^{50}$ G. Catalan, B. Noheda, J. McAneney, L. J. Sinnamon, and J. M. Gregg, Phys. Rev. B 72, 020102(R) (2005).

${ }^{51}$ H. N. Lee, H. M. Christen, M. F. Chisholm, C. M. Rouleau, and D. H. Lowndes, Nature (London) 433, 395 (2005). 